\title{
Reduction in invasive pneumococcal disease following implementation of the conjugate vaccine in the Oxfordshire region, England
}

\author{
Dona Foster, ${ }^{1,2}$ A. Sarah Walker, ${ }^{1}$ John Paul, ${ }^{2}$ David Griffiths, ${ }^{1}$ Kyle Knox, ${ }^{1}$ \\ Timothy E. Peto ${ }^{1}$ and Derrick W. Crook ${ }^{1}$ on behalf of the Oxford Invasive \\ Pneumococcal Surveillance Group \\ ${ }^{1}$ Nuffield Department of Medicine, University of Oxford, Oxford, UK \\ ${ }^{2}$ Health Protection Agency, Royal Sussex County Hospital, Brighton, UK
}

Correspondence

Dona Foster

dona.foster@ndm.ox.ac.uk

Derrick W. Crook

derrick.crook@ndcls.ox.ac.uk

Received 11 June 2010

Accepted 18 September 2010

\begin{abstract}
Pneumococcal conjugate vaccine to seven capsular types has been highly effective in the US since its introduction in 2000. The same vaccine was adopted by the UK in 2006. Ongoing surveillance since 1995 of invasive pneumococcal disease (IPD) in Oxfordshire, UK, allowed assessment of the impact of vaccine intervention. The vaccine significantly reduced IPD among the target group, children under 2 years of age; incidence rate ratio $(I R R)=0.62(95 \% \mathrm{Cl} 0.43-$ $0.90)(P=0.008)$ comparing the 3 years pre- and post-implementation with a residual incidence of 22.4/100 000 children. The reduction was even greater when comparing 11 years pre- with the 3 years post-implementation of vaccine; $\mathrm{IRR}=0.53(0.39-0.70)(P<0.0001)$. There was a marked direct effect of the vaccine evidenced by substantial reductions in the seven serotypes contained in the vaccine. There was also a clear reduction in IPD for those serotypes contained in the vaccine among those older than 2 years when comparing both the 3 and 11 year pre-PCV7 time periods, with IRR $=0.57(0.47-0.69)(P<0.0001)$ and $\mathrm{IRR}=0.50(0.43-0.58)(P<0.0001)$, respectively, indicating a strong herd effect. There was a significant, though moderate, rise in the serotypes not contained in the vaccine, with clear evidence for replacement in some serotypes.
\end{abstract}

\section{INTRODUCTION}

Streptococcus pneumoniae is a leading cause of infective morbidity and mortality worldwide (WHO, 2007). A protein-polysaccharide pneumococcal conjugate vaccine to only seven of the 92 known capsular types (PCV7) has been shown to be highly effective against invasive pneumococcal disease (IPD) (Whitney et al., 2003). Postimplementation observational studies in US populations showed major reductions in the burden of IPD for recipients and non-recipients of $\mathrm{PCV} 7$, indicating a strong direct and herd effect (Lexau et al., 2005; CDC, 2005; Pilishvili et al., 2010).

Based on the effectiveness of PCV7 in the US, PCV7 was added to the routine UK immunization schedule for children under 2 years of age in September 2006 (http://www.nhs. uk/Planners/vaccinations/Pages/Vaccinationchecklist.aspx). There were some differences between the US and UK that could, in theory, affect the impact of the vaccine. Pre-PCV7 implementation, the incidence of IPD (all ages) in the US was 24.3 per 100000 persons compared to 9.2 for the UK;

Abbreviations: $\mathrm{Cl}$, confidence interval; IPD, invasive pneumococcal disease; IRR, incidence rate ratio; NVT, non-vaccine serotype; VRT, vaccine-related serotype; $\mathrm{VT}$; vaccine serotype. while, in children under 5 years, it was reported as 98.7 in the US (calculated from reference CDC, 2008) compared to 24.3 in the UK (Foster et al., 2008). Furthermore, PCV7 would be expected to prevent at best $47 \%$ of IPD (all ages) in the UK compared to $62 \%$ in the US (Hausdorff et al., 2000a; Robinson et al., 2001; Foster et al., 2008) given the differences in serotypes causing IPD. A $3+1$ schedule (three primary doses followed by a booster) was recommended when the vaccine was introduced in the US in contrast to a reduced dose $(2+1)$ schedule later implemented in the UK and some other countries.

The impact of the PCV7 vaccine on a UK population of approximately 3 million residing around Oxford was investigated from an ongoing IPD surveillance project commenced in 1995.

\section{METHODS}

S. pneumoniae cases were identified from the Oxford Pneumococcal Surveillance Group study, covering a population of approximately 3 million people of all ages as previously published (Foster et al., 2008). IPD cases were defined by $S$. pneumoniae isolation from a normally sterile site [blood, cerebrospinal fluid or fluids (joint, pleural or ascitic)]. Date of birth, gender and diagnosis were recorded for each 
case. S. pneumoniae isolates were cultured and identified using standard microbiological techniques. All isolates were serotyped by the Quellung reaction using serotype-specific antiserum (Statens Serum Institut, Copenhagen, Denmark). This programme of enhanced surveillance has been ongoing under the same senior investigator's direction and without changes in surveillance methodology for 15 years (Sleeman et al., 2001; Foster et al., 2008). All statistical analysis was performed using Intercooled Stata 9 (StataCorp), with rates before and after PCV7 introduction compared using Poisson regression adjusted for the denominator population derived from the Office for National Statistics (http://www.statistics. gov.uk/downloads/theme_population/Population_trends_135.pdf).

\section{RESULTS AND DISCUSSION}

\section{Overall incidence of IPD}

A total of 3768 pneumococcal isolates were received during the 14-year study period (408 in under 2s): 3020 (355 in under $2 \mathrm{~s}$ ) isolates prior to PCV7 introduction and 748 (53 in under 2s) isolates post-PCV7. The mean incidence of IPD (all ages) in the Oxfordshire region of the UK in the 11 and 3 years prior to the introduction of PCV7 was $9.4(95 \% \mathrm{CI}$ 8.8-10.1) and 8.3 (95\% CI 7.9-8.8) per 100000 persons overall, respectively. The numbers and incidence of IPD by year are shown in Table 1. Post-PCV7, the mean incidence fell to 8.0 (CI 6.7-8.9) per 100000 persons, which was a significant decline in comparison with the 11 years prior to PCV7 [incidence rate ratio $(I R R)=0.80(95 \%$ CI 0.74-0.87), $P=0.001]$. When compared to the 3 years prior to PCV7, there was only a marginally significant decline in IPD $[$ IRR $=0.90$ (95\% CI 0.81-1.00), $P=0.06]$ (Table 2).

In children under 2 years of age, the target group for PCV7, the overall incidence in the 11 and 3 years prior to PCV7 was 43.3 (95\% CI 37.1-49.5) and 35.9 (95\% CI 26.9-41.2) per 100000 persons, respectively. This incidence has declined year on year: 29.5 (95\% CI 18.7-44.2), 24.0 (95\% CI 14.537.5 ) and 13.7 (95\% CI 6.8-24.5) with the mean being 22.4 (95\% CI 9.8-27.9) per 100000 persons following vaccine implementation (Table 1). Therefore, compared with 3 years post-PCV7, there has been a significant decline in the incidence of IPD over both 11 years [IRR $=0.53$ ( $95 \%$ CI $0.39-0.70), P<0.0001]$ and 3 years $[\mathrm{IRR}=0.62(95 \% \mathrm{CI}$ $0.43-0.90), P=0.008]$ prior to PCV7.

In those 2 years of age and over, there was no statistically significant evidence of change in incidence between the 3 years pre- versus post-PCV7 implementation [IRR $=0.93$ (95\% CI 0.84-1.04), $P=0.22$ ]; however, there was a significant decline compared to the 11 years pre-PCV7 when overall incidence was somewhat higher [IRR $=0.84$ (95\% CI 0.77-0.91), $P<0.0001]$. It should be noted that the incidence of IPD in the 2 years immediately prior to vaccine introduction was the lowest it had been over the whole pre-vaccine study period (see Table 1), a decline that probably reflects natural secular changes in the serotypes circulating in the region.

A significant decline in the burden of IPD among the target group, children under 2 years, for PCV7 was observed in this geographical area. The decline of at least $38 \%$ in the incidence of IPD was seen whether comparing 11 or 3 years pre-PCV7 with the 3 years post-PCV7. This is similar to that in other European reports, for example in the Netherlands (Rodenburg et al., 2010), but less than that reported for the US, where introduction of PCV7 in 2000 was followed by a $69 \%$ reduction in IPD in those under 2 years (Whitney et al., 2003). The total (all serotype) incidence among children under 2 years post-PCV7 in the Oxfordshire region was lower than that observed in the US 5 years post-PCV7 (22.4 compared to 38.9 per 100000 persons).

Table 1. Number of cases and incidence of IPD over the complete study period by age

PCV7 was introduced at the beginning of 2006-2007.

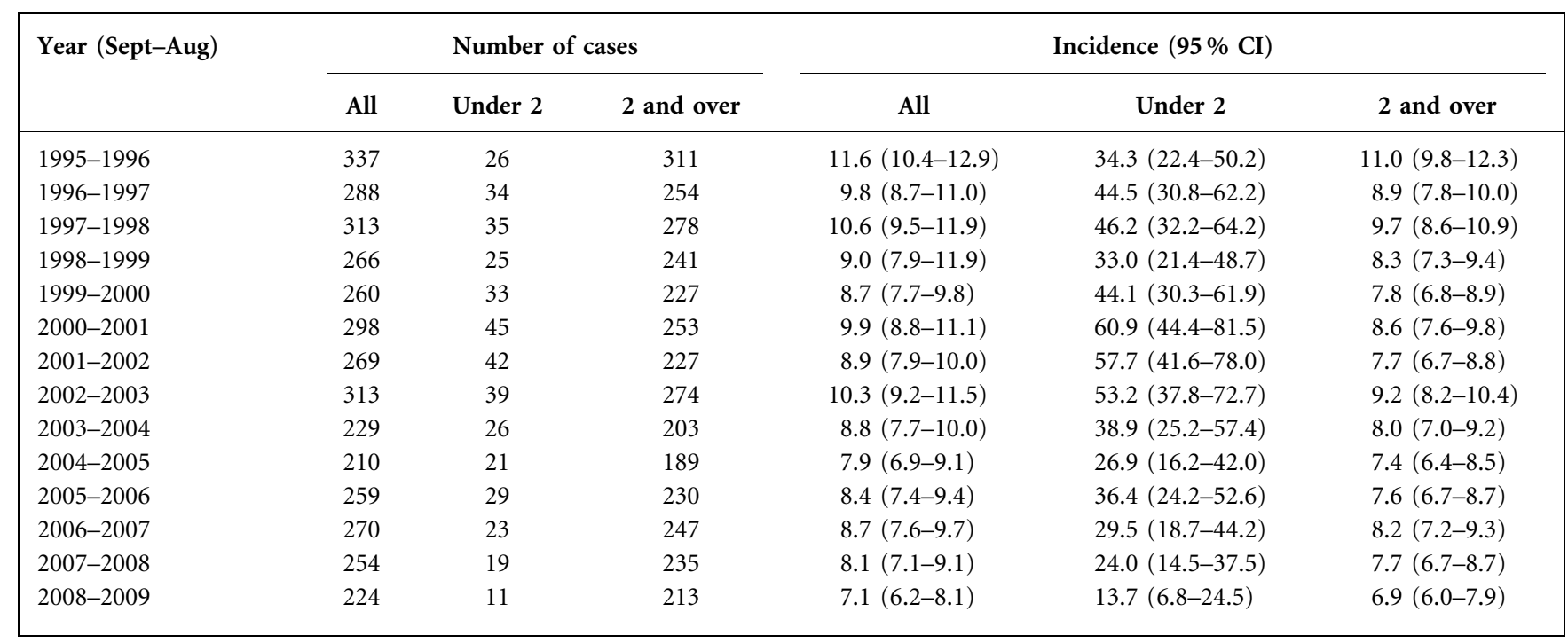


Table 2. IRR between incidence rate pre-PCV7 over 3 years compared to post-PCV7 over 3 years for the most frequent serotypes Incidences are per 100000 persons. VT, Vaccine serotype; VRT, vaccine-related type; NVT, non-vaccine type.

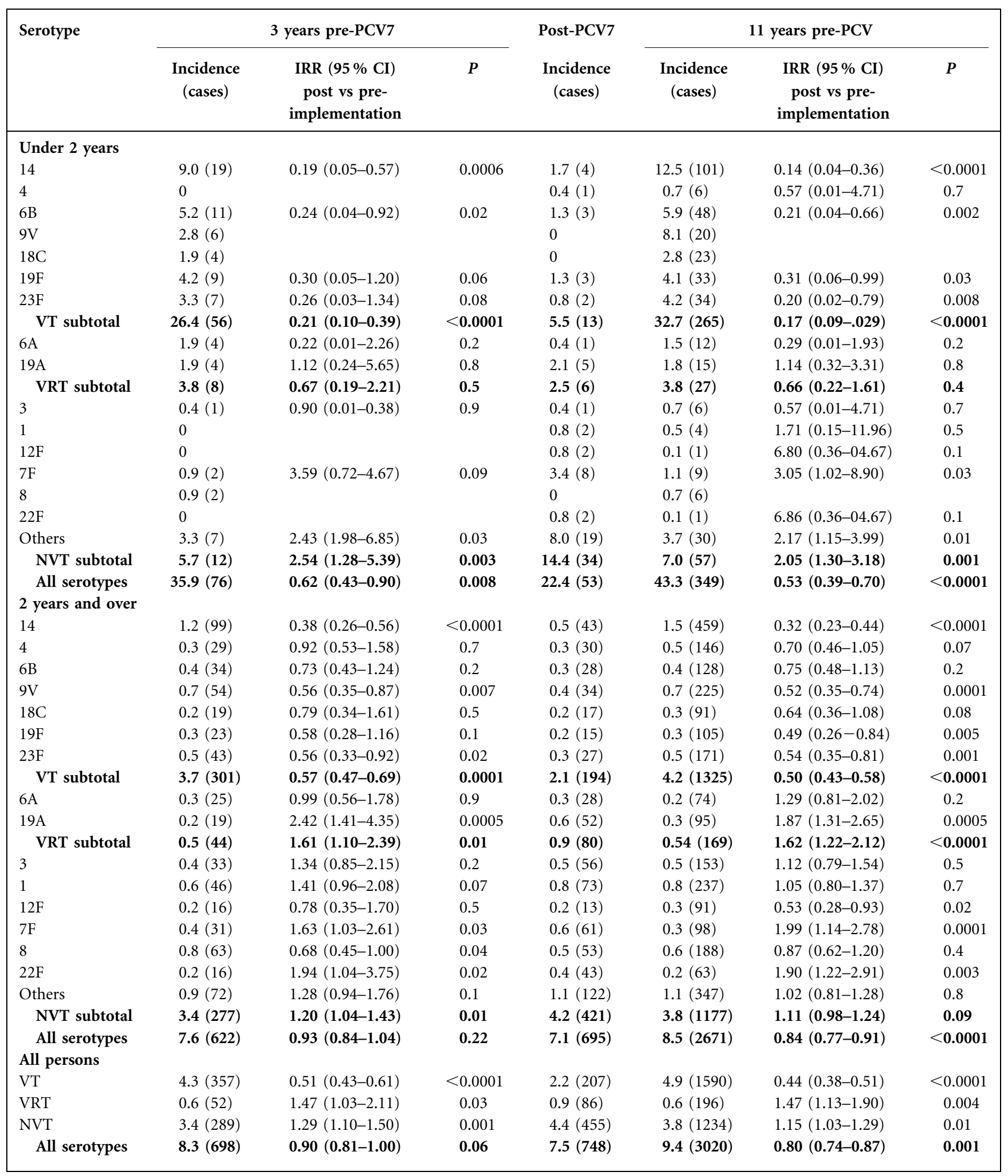




\section{Vaccine, vaccine-related and non-vaccine serotype incidence of IPD}

The serotypes contained in the PCV7 vaccine (serotypes 4, $6 \mathrm{~B}, 9 \mathrm{~V}, 14,18 \mathrm{C}, 19 \mathrm{~F}$ and $23 \mathrm{~F}$ ) were grouped together and termed vaccine serotypes (VTs) to compare incidence in the pre-vaccine era with that in the post-vaccine era. The vaccine-related serotypes (VRTs) are 19A and 6A. Nonvaccine serotypes (NVTs) are all the other serotypes detected in the study. The incidences of IPD caused by VTs, VRTs and NVTs are shown in Fig. 1 for those under 2 years and those 2 years and over. In those 2 years of age and over, there was a similar significant decline in VT IPD when comparing both pre-PCV7 time periods, with $\mathrm{IRR}=0.57 \quad(95 \% \quad$ CI $\quad 0.47-0.69) \quad(P<0.0001) \quad$ and $\mathrm{IRR}=0.50 \quad(95 \%$ CI $0.43-0.58) \quad(P<0.0001)$, respectively. However, in contrast, there was a significant increase in the VRTs $(P<0.01)$. There was a significant increase in NVT incidence post-PCV7 when the comparison was limited to the 3 years pre-implementation $(P=0.01)$, which was only of marginal significance when compared to the preceding 11 years $(P=0.09)$ (Table 2$)$.

In children under 2, there was a significant fall in VT IPD whether compared to 3 or 11 years pre-PCV $(P<0.0001)$ (Table 2). A significant rise in the incidence of NVTs also occurred compared to both pre-PCV time periods $(P<0.003)$. There was no significant change in VRTs $(P>0.3)$. The residual incidence of VT IPD in those under 2 years is declining steadily with a combined 3 year incidence of 5.5 (95\% CI 2.9-9.4), only slightly higher than the incidence 5 years post-PCV7 implementation in the US of 2.2 (calculated from reference CDC, 2008). The incidence of NVTs plus VRTs reported here of 16.9 (95\% CI 12.0-22.9) is substantially lower than the 36.7 reported in the US (calculated from reference CDC, 2008); however, this was associated with a twofold rise in NVTs in this region of the UK compared to only a $20 \%$ rise in nonPCV7 serotypes in the US. Crudely the rise in incidence seen in this study was substantially greater than observed in
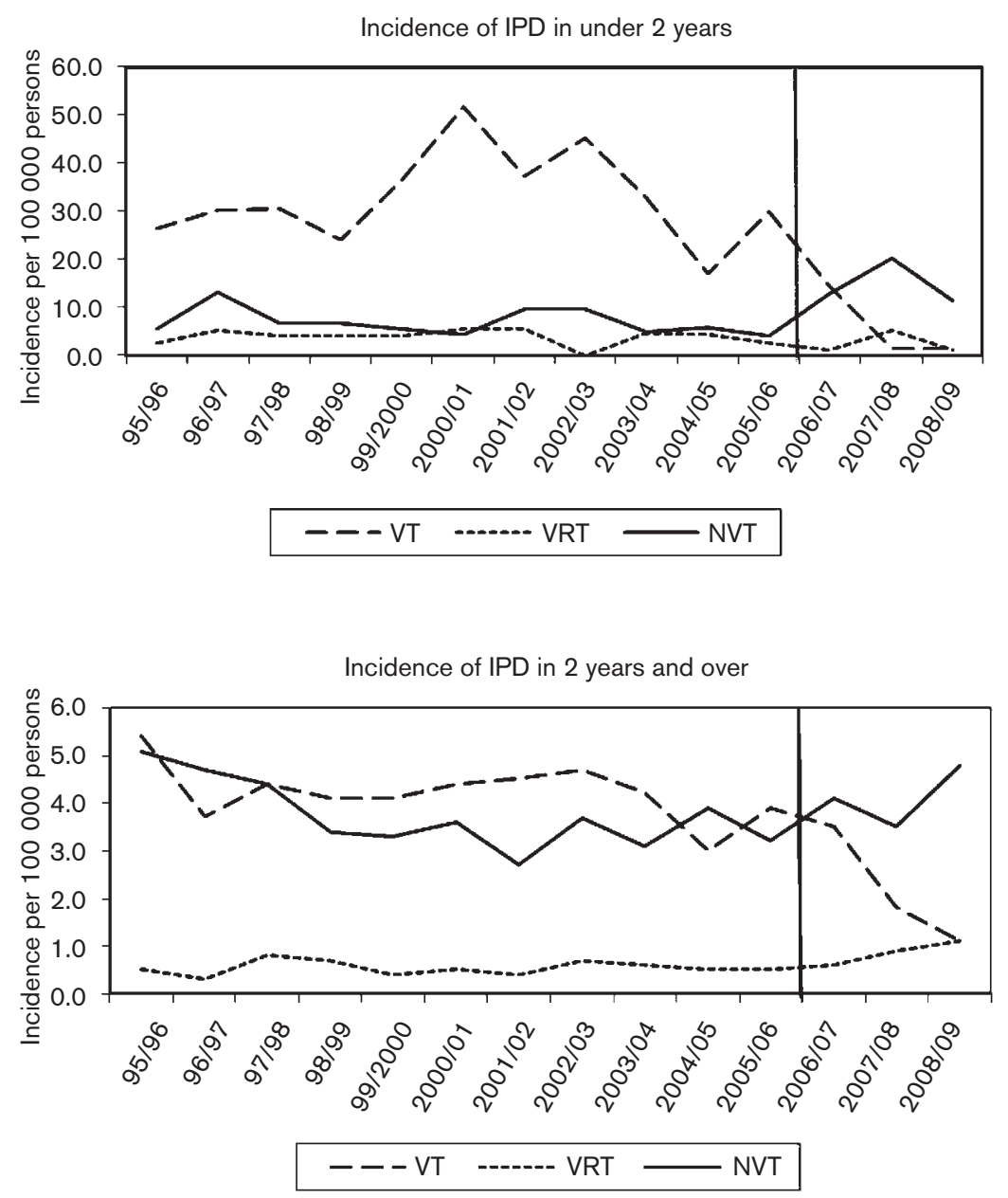

Fig. 1. Incidence of IPD over the study period in children under 2 years of age (top) and in those 2 years and older (bottom). VTs, vaccine serotypes (4,6B, 9V, 14, 18C, 19F, 23F); VRTs, vaccine-related types (19A, 6A); NVTs, non-vaccine types (all other serotypes). The vertical lines indicate vaccine introduction. 
the US for this age group, but, as numbers are relatively small and the overall level of NVTs and VRTs in the UK is still lower than in the US, it may be premature to regard this as an important difference. Nevertheless, the rise in NVTs is consistent with replacement (see below), which is well recognized with these pneumococcal vaccines. It is unclear yet whether this phenomenon will continue increasing in magnitude and will substantially reduce vaccine effectiveness.

A significant reduction of $40-50 \%$ in VT IPD was observed in those 2 years and over whether comparing 3 or 11 years pre-PCV7. This indicates an indirect or herd effect of the vaccine on older age groups. A modest rise in both NVTs and VRTs was observed, which diminished the overall reduction in the burden of IPD. There are no published data on the equivalent age categorization reported here for the US; however, for people over 50, the residual incidence reported here was calculated to be 14.7 per 100000 , which is substantially lower than that of 29.6 reported for the US (Lexau et al., 2005).

\section{Changes in IPD caused by individual serotypes}

The individual serotypes within PCV7 declined in both those 2 years and older and those under 2 years for both time periods, although their statistical significance varied by overall frequency, with serotype 4 showing the least decline (Table 2 and Fig. 2). In those over 2 years, serotypes 19A, 7F and $22 \mathrm{~F}$ increased significantly $(P<0.05)$ comparing both time periods pre-implementation to post-implementation: $19 \mathrm{~A}-\mathrm{IRR}=2.42(95 \%$ CI $1.41-4.35), P=0.0005$ (3 years) and IRR 1.87 (95\% CI 1.31-2.65), $P=0.0005$ ( 11 years); $7 \mathrm{~F}-$ $\mathrm{IRR}=1.63(95 \%$ CI $1.03-2.61), P=0.03 \quad$ (3 years) and
IRR $=1.99(95 \%$ CI $1.14-2.78), P=0.0001$ ( 11 years); and $22 \mathrm{~F}-\mathrm{IRR}=1.94(95 \% \mathrm{CI} 1.04-3.75), P=0.02$ ( 3 years $)$ and IRR $=1.90$ (95\% CI 1.22-2.91), $P=0.003$ (11 years) (Table 2 ). There were statistically significant rises in the incidence of serotypes $7 \mathrm{~F}$ and the 'other serotypes' in the under $2 \mathrm{~s}$ in both time periods [7F: IRR $=3.59$ (95\% CI 0.72-34.67), $P=0.09$ (3 years) and $\mathrm{IRR}=3.05$ (95\% CI $1.02-8.90)$, $P=0.03$ (11 years); 'other serotypes': IRR $=2.43(95 \% \mathrm{CI}$ 1.98-6.85), $P=0.03$ (3 years) and IRR $=2.17$ (95\% CI $1.15-$ 3.99), $P=0.01$ ( 11 years)].

Fig. 2 compares the annual year-on-year trend in incidence of each serotype pre- and post-PCV7 implementation (all ages). What is notable is that those serotypes increasing most substantially after PCV7 introduction (19A, 7F and $22 \mathrm{~F}$ ) were also increasing pre-PCV7, i.e. the change in relative risks suggest that the vaccine has likely had modest impact on enhancement of their population behaviour. Nevertheless, for 22F $(P<0.0001)$, 7F $(P<0.0001), 19 \mathrm{~A}$ $(P<0.0001)$ and 'other' serotypes $(P<0.0001)$, year-onyear increases post-implementation were significantly greater than year-on-year increases pre-implementation, providing direct evidence suggesting replacement. For serotypes 1 and 12, year-on-year decreases in incidence pre-implementation were halted $(12, P=0.8)$ or changed to significant increases $(1, P=0.003)$ post-implementation. In contrast, the seven VTs had modest, if any, year-on-year changes in incidence pre-PCV7, followed by substantial year-on-year declines.

\section{Resistance}

There was no evidence of change in penicillin resistance over the study period prior to the introduction of PCV7,

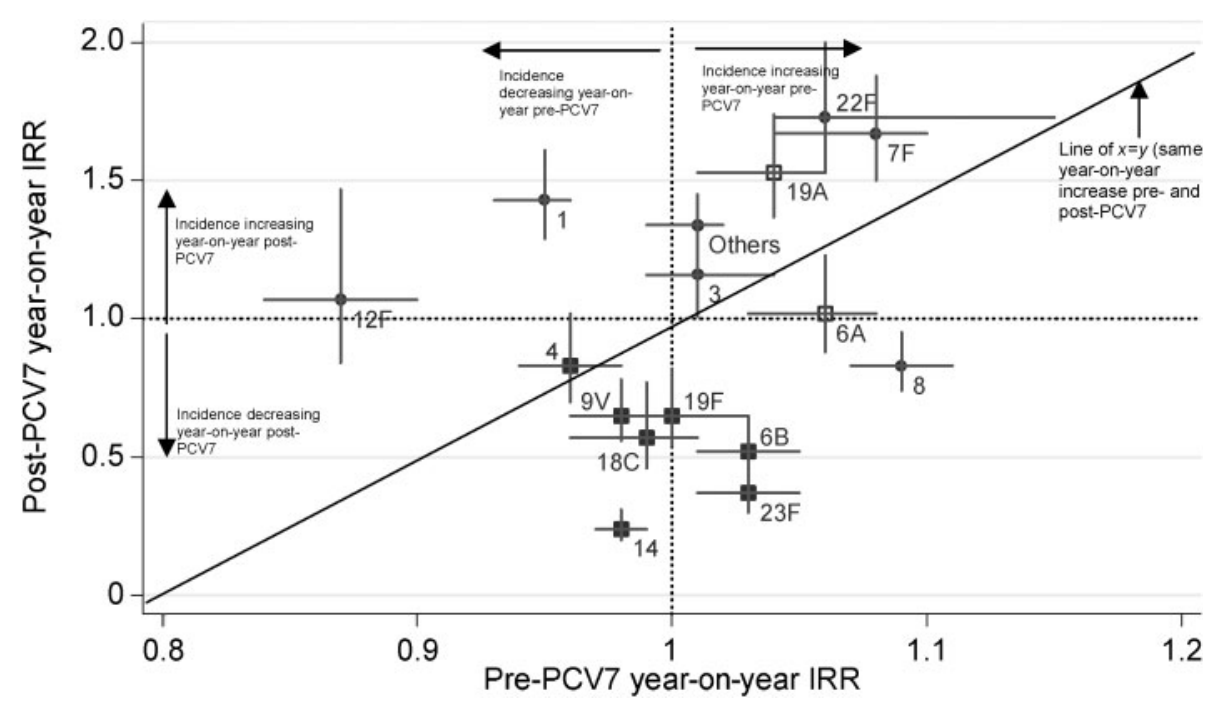

Fig. 2. IRR plot of serotypes 11 years pre- and 3 years post-PCV7 with 1 standard deviation using Poisson regression. The dotted line shows the IRR of 1 (no annual year-on-year change in incidence) for pre- and post-PCV7. $\mathbf{\square}$, VTs; $\square$, VRTs; $\bullet$, NVTs. 
the rate being constant at $5 \%$ of isolates. Penicillin resistance declined non-significantly after the introduction of PCV7 to $4.3 \%$ over the 3-year period. Erythromycin resistance fell from $13 \%$ pre-PCV7 to $5.5 \%$ post-PCV7, the fall associated mainly with the reduction in incidence of serotype 14. Post-PCV7, resistance to one or more antibiotics was detected in some VTs $(14,4,19 \mathrm{~F}$ and $6 \mathrm{~B})$ in 14 adult cases but no multiresistant isolates were detected from children under 2 years. The prevalence of antibiotic resistance in Oxfordshire remains low compared to that in other European countries (Beekmann et al., 2005) and the US.

Despite the study population being only 3 million, representing approximately $6 \%$ of the total population of England, it was sufficiently powered to significantly determine vaccine effects on IPD in all ages and for individual serotypes. These effects are in line with those reported by the Health Protection Agency for all of England and Wales (http://www.hpa.org.uk/web/HPAwebFile/HPAweb_ $\mathrm{C} / 1245581527892)$. Consequently, the observations reported here are likely to reflect the impact of the vaccine nationally for England and Wales. The magnitude of the reductions in the incidence of IPD reported here have not been as substantial as observed in the US. Much of the perceived difference in impact of the vaccine between the US and the UK may lie more with differences in the incidence of IPD prior to implementation of PCV7 in the two countries as the residual disease incidence is similar. The overall incidence in the US prior to introduction of PCV7 was higher at 23.2 cases per 100000 (Robinson et al., 2001) compared to 9.5 cases per 100000 (Foster et al., 2008). The difference in vaccine schedules, $2+1$ in the UK versus $3+1$ in the US, was another factor that potentially could have resulted in a difference in impact of the vaccine. However, comparable reductions in both pneumococcal carriage and invasive disease have been demonstrated for $3+1$ and reduced-dose schedules (van Gils et al., 2009). We also found little difference in the residual IPD caused by VTs in the US and the UK. Therefore, it does not seem likely that this difference in schedule is having a major effect on vaccine effectiveness.

Whether the level of residual disease following deployment of PCV7 will be similar wherever PCV7 is used worldwide remains to be seen, but the results of more vaccine programmes in both resource-limited and resource-rich countries will address this question.

In April 2010, the UK Department of Health introduced the 13-valent vaccine (PCV13) into the childhood vaccination programme in place of the 7 -valent vaccine. This vaccine offers protection against serotypes $1,5,7 \mathrm{~F}$, $19 \mathrm{~A}, 6 \mathrm{~A}$ and 3 in addition to those in the 7 -valent vaccine $(14,4,9 \mathrm{~V}, 19 \mathrm{~F}, 18 \mathrm{C}, 6 \mathrm{~B}$ and $23 \mathrm{~F})$. It has been shown to be immunogenic against all the serotypes and is well-tolerated (Scott et al., 2007). The potential serotype coverage in the Oxfordshire region over the whole study period for PCV13 compared to PCV7 is $84 \%$ versus $69 \%$, respectively, for the under 2 years age group and $72 \%$ compared to $45 \%$, respectively, in the 2 years and over. Similar issues to those reported here will inevitably relate to the ongoing implementation of PCV13, and follow-up surveillance of IPD after implementation will be required.

\section{ACKNOWLEDGEMENTS}

The surveillance project and D.F. were funded by Pfizer Pharmaceuticals (formally Wyeth Pharmaceuticals). The hospitals involved in establishing and maintaining the surveillance are as follows: Bedford, John Radcliffe, the Horton, Royal Berkshire, Stoke Mandeville, Wycombe, Kettering General, Northampton General, Wexham and Milton Keynes. D. F., A. S. W., D. G., T.E.P., J.P. and D.W.C. all participated in analysing the data and writing the paper. A. S. W., D. G., T.E.P. and D. W. C. are all funded by the NHS NIHR Oxford Biomedical Research Centre.

\section{REFERENCES}

Beekmann, S. E., Heilmann, K. P., Richter, S. S., Garcia-de-Lomas, J., Doern, G. V. \& GRASP Study Group (2005). Antimicrobial resistance in Streptococcus pneumoniae, Haemophilus influenzae, Moraxella catarrhalis and group A beta-haemolytic streptococci in 2002-2003. Results of the multinational GRASP Surveillance Program. Int $J$ Antimicrob Agents 25, 148-156.

CDC (2005). Direct and indirect effects of routine vaccination of children with 7-valent pneumococcal conjugate vaccine on incidence of invasive pneumococcal disease - United States, 1998-2003. MMWR Morb Mortal Wkly Rep 54, 893-897.

CDC (2008). Invasive pneumococcal disease in children 5 years after conjugate vaccine introduction - eight states, 1998-2005. MMWR Morb Mortal Wkly Rep 57, 144-148.

Foster, D., Knox, K., Walker, A. S., Griffiths, D. T., Moore, H., Haworth, E., Peto, T., Brueggemann, A. B. \& Crook, D. W. on behalf of the Oxford Invasive Pneumococcal Surveillance Group (2008). Invasive pneumococcal disease: epidemiology in children and adults prior to implementation of the conjugate vaccine in the Oxfordshire region, England. J Med Microbiol 57, 480-487.

Hausdorff, W. P., Bryant, J., Paradiso, P. R. \& Siber, G. R. (2000a). Which pneumococcal serogroups cause the most invasive disease: implications for conjugate vaccine formulation and use, part 1. Clin Infect Dis 30, 100-121.

Lexau, C. A., Lynfield, R., Danila, R., Pilishvili, T., Facklam, R., Farley, M. M., Harrison, L. H., Schaffner, W., Reingold, A. \& other authors (2005). Changing epidemiology of invasive pneumococcal disease among older adults in the era of pediatric pneumococcal conjugate vaccine. JAMA 294, 2043-2051.

Pilishvili, T., Lexau, C., Farley, M. M., Hadler, J., Harrison, L. H., Bennett, N. M., Reingold, A., Thomas, A., Schaffner, W. \& other authors (2010). Sustained reductions in invasive pneumococcal disease in the era of conjugate vaccine. J Infect Dis 201, 32-41.

Robinson, K. A., Baughman, W., Rothrock, G., Barrett, N. L., Pass, M., Lexau, C., Damaske, B., Stefonek, K., Barnes, B. \& other authors (2001). Epidemiology of invasive Streptococcus pneumoniae infections in the United States, 1995-1998. Opportunities for prevention in the conjugate vaccine era. JAMA 285, 1729-1735.

Rodenburg, G. D., de Greeff, S. C., Jansen, A. G., de Melker, H. E., Schouls, L. M., Hak, E., Spanjaard, L., Sanders, E. A. \& van der Ende, A. (2010). Effects of pneumococcal conjugate vaccine 2 years after its introduction, the Netherlands. Emerg Infect Dis 16, 816-823. 
Scott, D. A., Komjathy, S. F., Hu, B. T., Baker, S., Supan, L. A., Monahan, C. A., Gruber, W., Siber, G. R. \& Lockhart, S. P. (2007).

Phase 1 trial of a 13 -valent pneumococcal conjugate vaccine in healthy adults. Vaccine 25, 6164-6166.

Sleeman, K., Knox, K., George, R., Miller, E., Waight, P., Griffiths, D., Efstratiou, A., Broughton, K., Mayon-White, R. T. \& other authors (2001). Invasive pneumococcal disease in England and Wales: vaccination implications. J Infect Dis 183, 239-246.

van Gils, E. J., Veenhoven, R. H., Hak, E., Rodenburg, G. D., Bogaert, D., ljzerman, E. P., Bruin, J. P., van Alphen, L. \& Sanders, E. A. (2009).
Effect of reduced-dose schedules with 7-valent pneumococcal conjugate vaccine on nasopharyngeal pneumococcal carriage in children: a randomized controlled trial. JAMA 302, 159-167.

Whitney, C. G., Farley, M. M., Hadler, J., Harrison, L. H., Bennett, N. M., Lynfield, R., Reingold, A., Cieslak, P. R., Pilishvili, T. \& other authors (2003). Decline in invasive pneumococcal disease after the introduction of protein-polysaccharide conjugate vaccine. N Engl J Med 348, 17371746.

WHO (2007). Pneumococcal conjugate vaccine for childhood immunization - WHO position paper. Wkly Epidemiol Rec 82, 93-104. 